Original Research Paper

\title{
Could Geometry Demystify Patterns of Earth Quakes and Aftershocks? An Answer is Yes
}

\author{
Ramalingam Shanmugam \\ School of Health Administration, Texas State University, San Marcos, TX78666, USA
}

\author{
Article history \\ Received: 01-06-2015 \\ Revised: 11-03-2016 \\ Accepted: 15-03-2016 \\ Email: rs25@txstate.edu
}

\begin{abstract}
In this article, a pioneering (geometric) approach based on a new what is named here Glued Bivariate Poisson (GBP) distribution is constructed to demystify the 21 st century incidences of earthquakes and their aftershocks. A warning risk index for an earthquake or an aftershock to occur is formulated and computed for each of the thirteen major tectonic plates. This article reports a surprise and it is that there exists a geo angle constancy, 1.571 in the results pertaining to earthquakes or aftershocks in all the tectonic plates no matter what varying interlocked "glue level" among the African, Antarctica, Arabian, Australian, Caribbean, Coco's, Eurasian, Indian, North American, Pacific, Philippine, South American and Scotia tectonic plates. The contents of this article identified higher risk plates, which include Eurasian, India, Pacific and Philippines plates, where earthquakes of Kathmandu, Nepal (on 26 April 2015) and ChichShima, Japan (on 30 May 2015) occurred as validations of the contents of this article. An interesting geologic-constancy of 1.571 is noticed based on our statistical approach and an appropriate representative random sampling.
\end{abstract}

Keywords: Glued Bivariate Poisson Distribution, Prediction, Correlation, Regression

\section{Introduction}

The earliest earthquake occurred in $1831 \mathrm{BC}$ in the Shandong province of China. The world's deadliest earthquake occurred in $1556 \mathrm{AD}$ in central China and it killed 830,000 people. The largest earthquake in the world was a magnitude 9.5 in Chile on May 22, 1960. The largest earthquake so far in the United States (USA) occurred with a magnitude 9.2 in Prince William Sound, Alaska on Good Friday, March 28, 1964 UTC. In the year 2011 AD, Japan experienced an earthquake with a magnitude 9.0, which triggered a devastating tsunami. The Tsunami due to an earthquake in Sumatra island of Indonesia on December 26, 2004 killed at least 230,210 people. The 2008 Sichuan earthquake in China's Sichuan Province in May 2008 resulted in 69,227 fatalities. About 500,000 smaller or larger earthquakes are estimated to occur per year. Many geologists believe that earthquakes above magnitude 7.0 occur only every hundred years. However, there have been an average of 18 major earthquakes with magnitude 7.0 through 7.9 and mega earthquake with magnitude 8.0 or greater recently. The aftershocks much more than the earthquakes devastate public health killing more humans and destroying more buildings. Scientists wonder whether earthquakes and/or their aftershocks are more frequent in the 21 st century than in the past. If so, such a shift might be due advanced detection and more communication facilities. Let us look in the collected data in http://en.wikipedia.org.

First, let us learn the background in the prediction of earthquakes or aftershocks, which occur due to drifting tectonic plates (Wegener, 1912). What is tectonic plate? A German geologist Alfred Wegener announced the breakthrough concept of drifting plate tectonics in 1915 $\mathrm{AD}$ in his book The Origin of Continents and Oceans based on an observation that the east coast of South America and the west coast of Africa matched. According to Wegener, the lithosphere (the rigid outermost shell of earth), is broken up into eight major tectonic plates and many minor plates. Where the plates meet, their relative motion determines the boundary, which are convergent, divergent, or transform type. Figure 1 for a view of 13 major tectonic plates.

What is an aftershock? An aftershock is a tremor with a smaller magnitude, which occurs after an earthquake. If the magnitude of an aftershock is larger than the main earthquake, the aftershock is redesignated as the main shock and the original main shock is re-designated as a foreshock. 


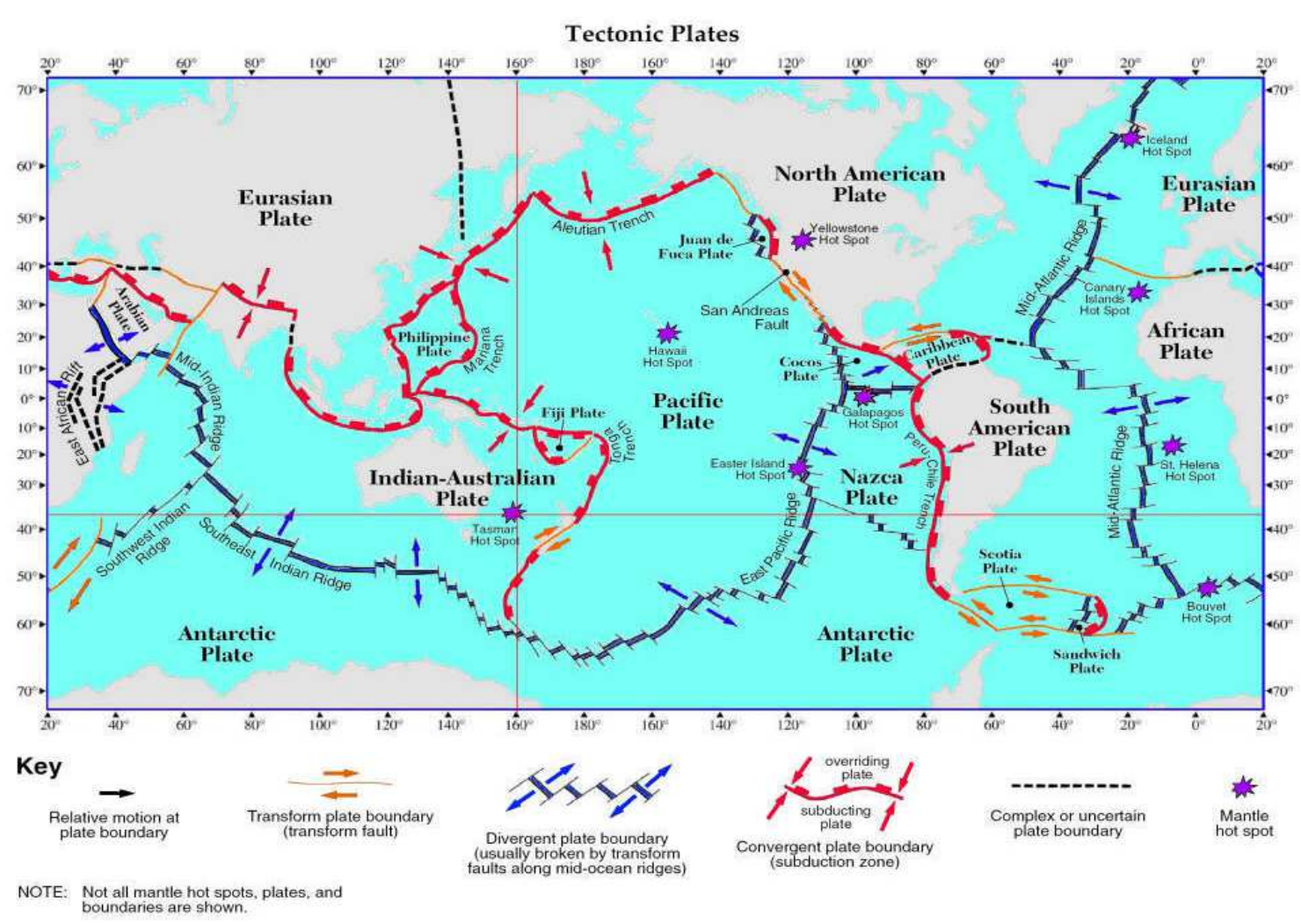

Fig. 1. World map of tectonic plates (http://www.unavco.org/software/geodetic-utilities/plate-motion-calculator/plate-motioncalculator.html)

How much damage an earthquake or aftershock can make? To comprehend it, a chronological order of literature helps. Richter (1958) gave details of elementary seismology. Ogata (2001) proved that probability of large earthquakes increased near aftershock regions with relative quiescence. Polat et al. (2002) analyzed and interpreted the aftershocks sequence in Izmit (Turkey) earthquake. Rubin (2002) probed the aftershocks of micro earthquakes as the mechanics of rupture. Shcherbakov and Turcotte (2004) investigated the damage mechanics of the aftershocks.

Shcherbakov et al. (2005) compiled the aftershock statistics and searched for clues in them. Helmstetter et al. (2005) summarizes the importance and role of small earthquakes for stress transfers and earthquake triggering. Shcherbakov et al. (2006) computed the correlations in aftershock and seismicity patterns and advocated the importance of aftershocks to predict the future earthquake. Felzer and Brodsky (2006) noted that aftershock density decays with distance indicates triggering by dynamic stresses. Abe and Suzuki (2007) used the correlation to relate seismicity with aftershocks.

Schoenberg et al. (2008) provide the geometric view of the distribution of Voronoi cells generated stress. Métivier et al. (2009) announced the evidence of earthquake as triggered by the solid earth tides.
Shearer and Stark (2012) noted that the global risk of big earthquake has not recently increased but has been for a while. Beroza (2012) came up with a formula to estimate about how many great earthquakes should we expect. Marekova (2014) provided an analysis of the spatial distribution between successive earthquakes in aftershock series.

To visualize the relationship between the number, $\mathrm{X}$ of the earthquakes over the magnitude 6.0 and the number, $\mathrm{Y}$ of the aftershocks below the magnitude 6.0 in Rector Scale, let us consider Ogata (2001) data of in a micro region with longitude interval (130-152 $)$ and the latitude interval $\left(27-45^{\circ}\right)$ in Table 1 . A preliminary analysis offers a clue to develop a suitable underlying model for the data as their correlation is $\rho_{(x, y)}=0.986$. The configuration in Fig. 2 suggests that more earthquakes with magnitude higher than 6.0 in Rector Scale implies more aftershocks that are frequent. A reason might be that the adjacent tectonic plates break loose from their "glues". The "glue level" ought to be factor in the modeling of earthquakes and aftershocks data. The glue level is not directly observable but could be accommodated, for the first time, as a parameter of the underlying model and it is exactly done in this article. 


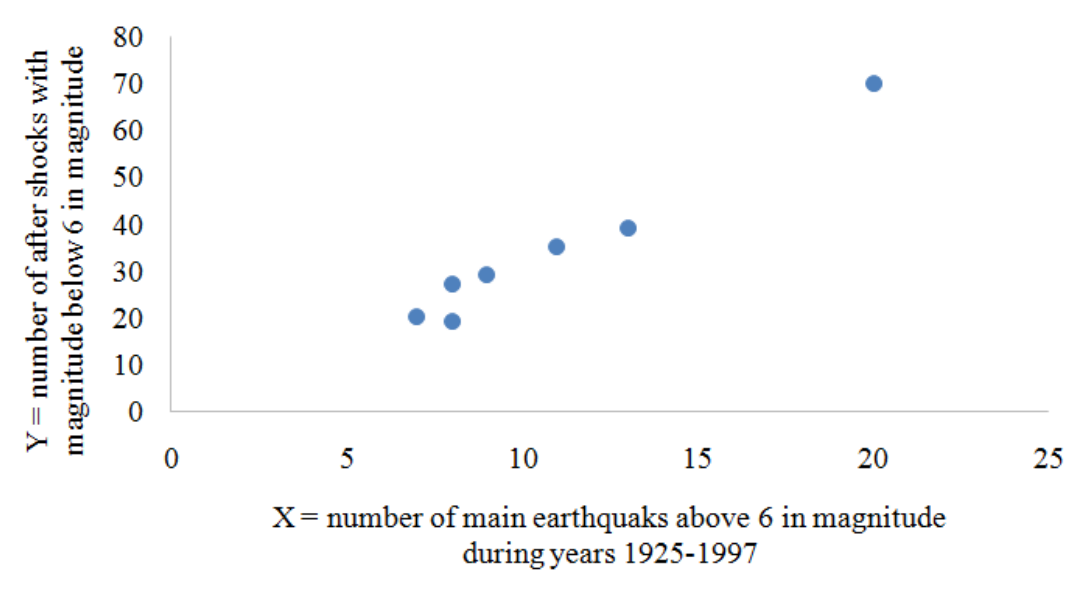

Fig. 2. Glued X, number of earthquakes above 6 magnitude and Y, number of aftershocks

Table 1. Ogata (2001, $\mathrm{X}=\#$ of shocks $>6$ magnitude and $\mathrm{Y}=\#$ of aftershocks $<6$ magnitude within specified micro region with longitude and latitude intervals during years 1925-1997

\begin{tabular}{lllll}
\hline $\begin{array}{l}\text { Regional area } \\
\text { (longitude, latitude) }\end{array}$ & $\begin{array}{l}\text { Duration } \\
\text { (year 1, year 2) }\end{array}$ & $\begin{array}{l}\mathrm{X}=\text { number of main } \\
\text { earthquakes Above 6.0 }\end{array}$ & $\begin{array}{l}\text { Y=number of } \\
\text { aftershocks Below 6.0 }\end{array}$ & $\begin{array}{l}\text { Range of aftershock } \\
\text { magnitude (min, max) } \\
\text { In rector scale }\end{array}$ \\
\hline A (142-151, 41-45) & $(1982,1995)$ & 9 & 29 & $(3.3,8.2)$ \\
B (140-144, 37-41) & $(1928,1996)$ & 20 & 70 & $(0.0,6.9)$ \\
C $(137-140,37-42)$ & $(1939-1995)$ & 7 & 20 & $(0.0,5.3)$ \\
D $(139-142,33-37)$ & $(1931-1990)$ & 11 & 35 & $(0.0,5.5)$ \\
E $(135-139,35-37)$ & $(1941-1984)$ & 8 & 19 & $(0.0,4.7)$ \\
F $(134-137,33-36)$ & $(1925-1995)$ & 8 & 27 & $(0.0,5.5)$ \\
G $(130-135,27-36)$ & $(1943-1997)$ & 13 & 39 & $(0.0,5.0)$ \\
Correlation $\rho_{(x, y)}$ & & 0.986 & &
\end{tabular}

To elaborate this new line of research thinking, a Glued Bivariate Poisson (GBP) distribution with its statistical properties and a geometric methodology based on GBP are introduced in this article. The conceptual and methodological contributions are illustrated using the incidences of earthquake and aftershock in the 21st century in 13 tectonic plates (they are African, Antarctica, Arabian, Australian, Caribbean, Coco's, Eurasian, Indian, North American, Pacific, Philippine, South American and Scotia plates). The genius and Nobel laureate Albert Einstein established the fundamental property $E=M C^{2}$ where $E, M$ and $C$ denote respectively the energy, mass and speed of light respectively. Likewise, this article finds a surprise and it is that there exists a geo angle constancy, 1.571 in the results pertaining to earthquakes and aftershocks in all the tectonic plates no matter what varying interlocked "glue level" among them.

\section{Derivation of Glued Bivariate Distribution and its Properties}

To be specific, let $X \geq 0$ and $Y \geq 0$ be the number of earthquakes and aftershocks in a tectonic plate with a "geologic glue level" $\phi \geq 0$ with its adjacent plates, during a time with incidence rate $\lambda_{1}>0$ and $\lambda_{2}>0$ respectively. The earth functions with a chance mechanism, which generates both $X$ and $Y$. This article proposes a candidate to be the underlying distribution for the Random Variable (RV) $X$ and $Y$. It is:

$$
\begin{aligned}
& p\left(x, y \mid \lambda_{1}, \lambda_{2}, \phi\right) \\
& =\left[\frac{(1+\phi x y)}{\left(1+\phi \lambda_{1} \lambda_{2}\right)}\right]\left[e^{-\left(\lambda_{1}+\lambda_{2}\right)} \lambda_{1}^{x} \lambda_{2}^{y} / x ! y !\right] ; \\
& x, y=0,1,2, \ldots, \infty ; \lambda_{1} \geq 0, \lambda_{2} \geq 0, \phi \geq 0 .
\end{aligned}
$$

The Probability Mass Function (PMF) in (1) is named "glued bivariate Poisson" distribution. The factor $1 /\left(1+\phi \lambda_{1} \lambda_{2}\right)$ is a normalizing constant. Berkhout and Plug (2004) studied a different bivariate Poisson distribution for count data in general.

When the "geologic glue" is negligible (that is, $\phi=$ 0 ), the GBP distribution (1) reduces to the product of their marginal probability mass functions $p\left(x \mid \lambda_{1}\right)=e^{-\lambda_{1}} \lambda_{1}^{x} / x$ ! and $p\left(y \mid \lambda_{2}\right)=e^{-\lambda_{2}} \lambda_{2}^{y} / y$ !, implying that the occurrence of an aftershocks is independent of the 
occurrence of its earthquakes. If it is the case, the configuration in Fig. 2 ought to have no visible pattern but it did not happen so. Otherwise (that is, $\phi \neq 0$ ), the occurrence of earthquake is correlated with the occurrence of its aftershocks. This issue needs to be sorted out.

To sort out, we note that:

$$
\begin{aligned}
& E(X Y)=\sum_{x=0}^{\infty} \sum_{y=0}^{\infty} p\left(x, y \mid \lambda_{1}, \lambda_{2}, \phi\right) \\
& =\frac{\lambda_{1} \lambda_{2}\left[1+\phi\left(1+\lambda_{1}\right)\left(1+\lambda_{2}\right)\right]}{\left(1+\phi \lambda_{1} \lambda_{2}\right)}
\end{aligned}
$$

The product moment, $E(X Y)$ is the product, $\lambda_{1} \lambda_{2}$ of the rate of incidences of the earthquakes and aftershocks, when the tectonic plates are not geologically glued or with a weaker geologic glue level (that is, $\phi=0$ ). Otherwise (that is, with a stronger geologic glue level $\phi$ $\neq 0)$, the product moment, $E(X Y)$ increases, proportionally to $\frac{\left[1+\phi\left(1+\lambda_{1}\right)\left(1+\lambda_{2}\right)\right]}{\left(1+\phi \lambda_{1} \lambda_{2}\right)}$. The marginal PMF of the number of earthquakes is:

$$
\begin{aligned}
& p\left(x \mid \lambda_{1}, \phi\right)=\sum_{y=0}^{\infty}\left[\frac{(1+\phi x y)}{\left(1+\phi \lambda_{1} \lambda_{2}\right)}\right]\left[e^{-\left(\lambda_{1}+\lambda_{2}\right)} \lambda_{1}^{x} \lambda_{2}^{y} / x ! y !\right] \\
& =\left[\frac{\left(1+\phi \lambda_{2} x\right)}{\left(1+\phi \lambda_{1} \lambda_{2}\right)}\right] e^{-\lambda_{1}} \lambda_{1}^{x} / x ! \\
& x=0,1,2, \ldots, \infty ; \lambda_{1} \geq 0, \phi \geq 0 .
\end{aligned}
$$

The expected value and the variance of the number of earthquakes are respectively:

$$
\begin{aligned}
& E\left(X \mid \lambda_{1}, \phi\right)=\sum_{x=0}^{\infty} x p\left(x \mid \lambda_{1}, \phi\right) \\
& =\left[1+\phi\left(1+\lambda_{1}\right) \lambda_{2}\right]\left(\frac{\lambda_{1}}{\left(1+\phi \lambda_{1} \lambda_{2}\right)}\right)
\end{aligned}
$$

The Poisson chance mechanism is known to keep no memory of the past patterns and the incidences are rare and sporadic. Johnson et al. (1997) for narration of different Poisson chance mechanisms. Likewise, there exists a parallelism with respect to the number of aftershocks. The marginal PMF of the number of aftershocks is:

$$
\begin{aligned}
& p\left(y \mid \lambda_{2}, \phi\right)=\sum_{x=0}^{\infty}\left[\frac{(1+\phi x y)}{\left(1+\phi \lambda_{1} \lambda_{2}\right)}\right]\left[e^{-\left(\lambda_{1}+\lambda_{2}\right)} \lambda_{1}^{x} \lambda_{2}^{y} / x ! y !\right] \\
& =\left[\frac{\left(1+\phi \lambda_{1} y\right)}{\left(1+\phi \lambda_{1} \lambda_{2}\right)}\right] e^{-\lambda_{2}} \lambda_{2}^{y} / y ! \\
& y=0,1,2, \ldots, \infty ; \lambda_{2} \geq 0, \phi \geq 0 .
\end{aligned}
$$

The expected value and the variance of the number of aftershocks are respectively:

$$
\begin{aligned}
& E\left(Y \mid \lambda_{2}, \phi\right)=\sum_{y=0}^{\infty} y p\left(y \mid \lambda_{2}, \phi\right) \\
& =\left[1+\phi \lambda_{1}\left(1+\lambda_{2}\right)\right]\left(\frac{\lambda_{2}}{\left(1+\phi \lambda_{1} \lambda_{2}\right)}\right)
\end{aligned}
$$

In other words, the role of glue among the tectonics is clear. However, the covariance between the number, $X$ of earthquakes and the number, $Y$ of aftershocks is:

$$
\begin{aligned}
& \operatorname{Cov}(X, Y) \\
& =E(X Y)-E(X) E(Y) \\
& =\frac{\phi \lambda_{1} \lambda_{2}}{\left(1+\phi \lambda_{1} \lambda_{2}\right)^{2}}
\end{aligned}
$$

Using the product moment, $E(X Y)$ in (2), the marginal moments $E(X)$ in (4) and $E(Y)$ in (7). The $C o v$ $(X, Y)$ in (7) is zero when the tectonic plates are not glued or weakly glued (that is, $\phi=0$ ). The covariance increases with a stronger glue level among the tectonic plates. Next, the estimator of the GBP parameters $\left(\lambda_{1}, \lambda_{2}\right.$, $\phi)$ with a bivariate random sample $\left(x_{1}, y_{1}\right),\left(x_{2}, y_{2}\right), \ldots .$. , $\left(x_{n}, y_{n}\right)$ of size $n \geq 2$ from the GBP in (1). They are:

$$
\begin{aligned}
& \hat{\lambda}_{1, \hat{\phi}} \approx \bar{x}(1+\hat{\phi} \bar{y}) \\
& \hat{\lambda}_{2, \hat{\phi}} \approx \bar{y}(1+\hat{\phi} \bar{x})
\end{aligned}
$$

And:

$$
\begin{aligned}
& \hat{\phi} \approx\left|\frac{\gamma_{x, y}}{\hat{\lambda}_{1} \hat{\lambda}_{2}\left[\left(1+\hat{\lambda}_{1}\right)\left(1+\hat{\lambda}_{2}\right)-\gamma_{x, y}\right]}\right| \\
& =\left|\frac{\sum_{i=1}^{n}\left(x_{i} y_{i}-\overline{x y}\right)}{\overline{x y}\left[(1+\bar{x})(1+\bar{y})-\sum_{i=1}^{n}\left(x_{i} y_{i}-\overline{x y}\right)\right]}\right|
\end{aligned}
$$

where, $\gamma_{x, y}$ denotes the sample covariance of between $x$ and $y$. When the covariance, $\gamma_{x, y}=0$ between the number of earthquakes and the number of aftershocks in a data, the estimate, $\hat{\phi}$ of the glue level among the tectonic plates is zero. Consequently, the incidence rate of earthquakes and aftershocks are respectively the mean $\bar{x}$ and $\bar{y}$. Otherwise (that is, when $\phi \neq 0$ ), the incidence rate of earthquakes and aftershocks increase respectively by a factor of $(1+\hat{\phi} \bar{y})$ and $(1+\hat{\phi} \bar{x})$. This article pursues a geometric line of thinking to demystify the uncertainty with respect to the incidences of earthquakes and aftershocks. In the Fig. 3 for the geometric description, the points $O:\left(o_{1}, o_{2}\right)=(0,0)$ and $U:\left(u_{1}, u_{2}\right)=(1,1)$ denote respectively the location of origin and the location of completion for the probabilities. 


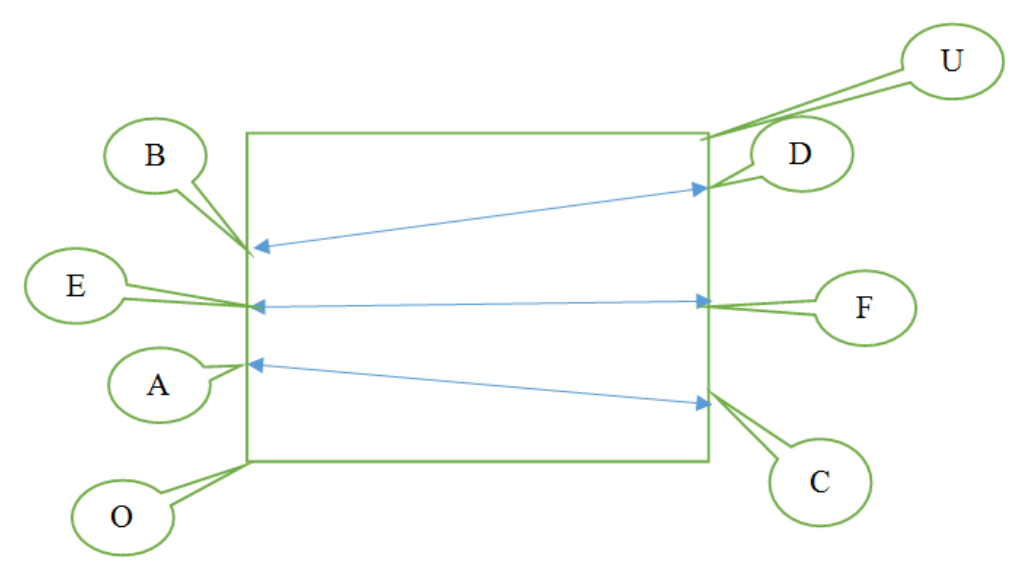

Fig. 3. Trapezoidal chances among no and one earthquake versus no and one aftershock

Let the points $A:\left(a_{1}, a_{2}\right)=\left(0, p_{x, 0}\right), B:\left(b_{1}, b_{2}\right)=\left(0, p_{y, 0}\right)$, $C:\left(c_{1}, c_{2}\right)=\left(0, p_{x, 1}\right)$ and $D:\left(d_{1}, d_{2}\right)=\left(0, p_{y, 0}\right)$ denote the vectors in two dimensional Cartesian coordinates in a mapping (Fig. 3), where:

$$
\begin{aligned}
& p_{x, 0}=\operatorname{Pr}(X=0)=\frac{e^{-\lambda_{1}}}{\left(1+\phi \lambda_{1} \lambda_{2}\right)} \\
& p_{y, 0}=\operatorname{Pr}(Y=0)=\frac{e^{-\lambda 2}}{\left(1+\phi \lambda_{1} \lambda_{2}\right)} \\
& p_{x, 1}=\operatorname{Pr}(X=1)=\frac{\lambda_{1} e^{-\lambda_{1}}}{\left(1+\phi \lambda_{1} \lambda_{2}\right)}
\end{aligned}
$$

And:

$$
p_{y, 1}=\operatorname{Pr}(Y=1)=\frac{\lambda_{2} e^{-\lambda 2}}{\left(1+\phi \lambda_{1} \lambda_{2}\right)}
$$

First, note that $\mathrm{AB}$ is parallel to $\mathrm{CD}$. Hence, the configuration $\mathrm{ABCD}$ is trapezoid and it could be rectangle if the vector length $\mathrm{BD}$ is equal to the vector length $\mathrm{AC}$. In addition, if the vector length $\mathrm{AB}$ is equal to the vector length $\mathrm{AC}$, the configuration $\mathrm{ABCD}$ is square.

The vector length $A B=\frac{\left(a_{1} b_{1}+a_{2} b_{2}\right)}{\sqrt{\left(a_{1}^{2}+a_{2}^{2}\right)\left(b_{1}^{2}+b_{2}^{2}\right)}}$ denotes a gap between the chances of no earthquake and no aftershocks and it is a unit vector (that is, $A B=1$ ). In other words, the vector length $\mathrm{AB}$ designates an index of safe period.

The vector length $C D=\frac{\left(1+p_{x, 1} p_{y, 1}\right)}{\sqrt{\left(1+p_{x .1}^{2}\right)\left(1+p_{y, 1}^{2}\right)}}$, which is clearly not equal to the unit vector $A B$, unless $p_{x, 1}=p_{y, 1}$ or equivalently there is a constancy of the earthquake rate and aftershock rate (that is, $\lambda_{1}=\lambda_{2}=$ cons tan $t$ ).
What does the constancy of the earthquake rate and aftershock rate mean in geological sense? All it means is that the rate of the rare Poisson nature of earthquake events is adapted intact with respect to the rate of tectonic plate's breakup as aftershocks. Does it happen this way in real life? We will examine it in Section 3 with the actual incidences of 21 st century.

Furthermore, the ratio $\Re_{e q}=\frac{C D}{A B}$ indicates an index level of dilution of the constancy of the earthquake rate. A higher value of the index level of dilution means a wider gap between the chances of an earthquake and an aftershock (an alarming period of spectrum) in comparison to the gap between the chances of no earthquake and no aftershock (a safe period of spectrum).

The vector length $A C=\frac{\left(a_{1} c_{1}+a_{2} c_{2}\right)}{\sqrt{\left(a_{1}^{2}+a_{2}^{2}\right)\left(c_{1}^{2}+c_{2}^{2}\right)}}$ signifies the risk of earthquake exiting the comfort zone of no earthquake to uncomfortable zone of experiencing an earthquake and it is $A C=\frac{p_{x, 1}}{\sqrt{\left(1+p_{x .1}^{2}\right)}}$. The vector length $A C$ equals the vector length $B C$. The angle $\operatorname{Cos}(\angle A C B)=\frac{1}{2}\left(1-\frac{1}{A C^{2}}\right)$. Consequently, the angle of an earthquake incidence is:

$\left.\theta_{e q}=\angle A C B\right)=\operatorname{Cos}^{-1}\left\{\frac{1}{2}\left(1-\frac{1}{A C^{2}}\right)\right\}$

Likewise, the vector length $B D=\frac{\left(b_{1} d_{1}+b_{2} d_{2}\right)}{\sqrt{\left(b_{1}^{2}+b_{2}^{2}\right)\left(d_{1}^{2}+d_{2}^{2}\right)}}$ signifies the risk of aftershock exiting the comfort zone of no aftershock to uncomfortable zone of experiencing an aftershock and it is $B D=\frac{p_{y, 1}}{\sqrt{\left(1+p_{y .1}^{2}\right)}}$. The vector length $A D$ equals the 
vector length $B D$. The angle $\operatorname{Cos}(\angle A D B)=\frac{1}{2}\left(1-\frac{1}{B D^{2}}\right)$. Consequently, the angle of an earthquake incidence is:

$$
\left.\theta_{a s}=\angle A D B\right)=\operatorname{Cos}^{-1}\left\{\frac{1}{2}\left(1-\frac{1}{B D^{2}}\right)\right\}
$$

The ratio $\mathfrak{R}_{a s}=\frac{B D}{A C}$ indicates an index level of dilution of the constancy of the aftershock rate. A higher value of the index level of dilution means a wider gap between the chances of an earthquake and an aftershock (an alarming period of spectrum) in comparison to the gap between the chances of no earthquake and no aftershock (a safe period of spectrum).

To compare and interpret how the thirteen tectonic plates behave with respect to the risk of both earthquakes and aftershocks, a percent risk index:

$$
\mathfrak{I}=100\left(\frac{\mathfrak{R}_{e q}}{1+\mathfrak{R}_{e q}}\right)\left(\frac{\mathfrak{R}_{a s}}{1+\mathfrak{R}_{a s}}\right)
$$

Is worthwhile to compute and display. Larger the risk index $\mathfrak{I}$ means a higher warning for either earthquake or aftershock or both.

\section{Illustration of 21st Century Earthquake Incidences and Warnings to Avoid Future Devastations}

In this section, the geometric concept to capture and interpret a combined risk level for an earthquake or aftershock in thirteen tectonic plates on earth based on what this article named "glue level" among the tectonic plates. The names of the thirteen tectonic plates are Antarctica, Arabian, Australia, Caribbean, Cocos, Eurasian, Indian, North American, Pacific, Philippines,
South American and Scotia. The number, $x$ of earthquakes and the number, $y$ aftershocks are aggregate of what happened in each major plate and its interlocked adjacent plates. Figure 1 to visualize the names of the adjacent plates for each among the thirteen major plates. All the derived expressions in section 2, based on glued bivariate Poisson distribution are illustrated using earthquakes $(x)$ and aftershocks $(y)$ incidences during 21 st century (see their summary in Table 2 and original data in the webpage http://en.wikipedia.org.

It is clear (see $\rho_{(x, y)}$ Table 2 or the configuration in Fig. 4) that the number of earthquakes and number of aftershocks are correlated. This knowledge suggests that the plates must have been interlocked with some sort of "glue" which would break loose the adjacent plates when an earthquake or aftershock occurs in one plate causing chain reactions. The understanding of the mystery behind the pattern of earthquake or aftershock could be enhanced with a quantified knowledge of the "glue level", $\phi \geq 0$. Using the 21 st century data on earthquakes and aftershocks along with the GBP distribution (1) and the estimator (10), the estimates of the "glue" level are obtained and displayed in Table 2. Smaller the "glue" level refers a stronger interlocking among the plates. The "glue" level ranged from 0.01 (in African, Arabian, Indian, North American, Pacific, Philippine plates) to 0.65 (in Scotia plate). The "glue" levels of Australian, Eurasian, Caribbean and South American plates are intermediary.

Using the 21 st century data, the estimators (8) and (9), the rate of earthquakes and the aftershocks are obtained, displayed in Table 2 and graphed individually in Fig. 5 and 6 respectively. Clearly, the incidence of earthquake as well as the aftershock declined as the "glue" level is higher. The glue level signifies how strongly the plates are geologically interlocked.

Among 13 tectonic plates

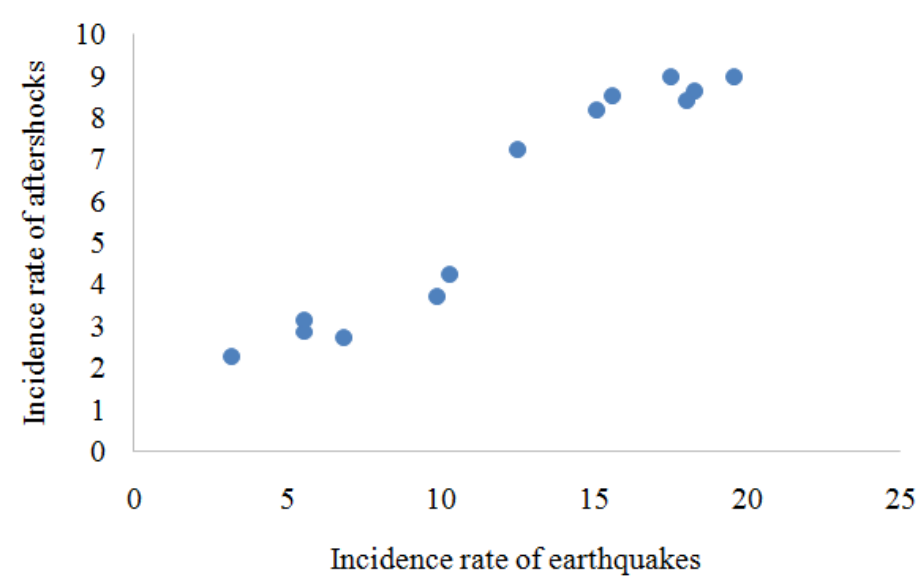

Fig. 4. Pattern between earthquakes and aftershocks 
Comparison of 13 tectonic plates

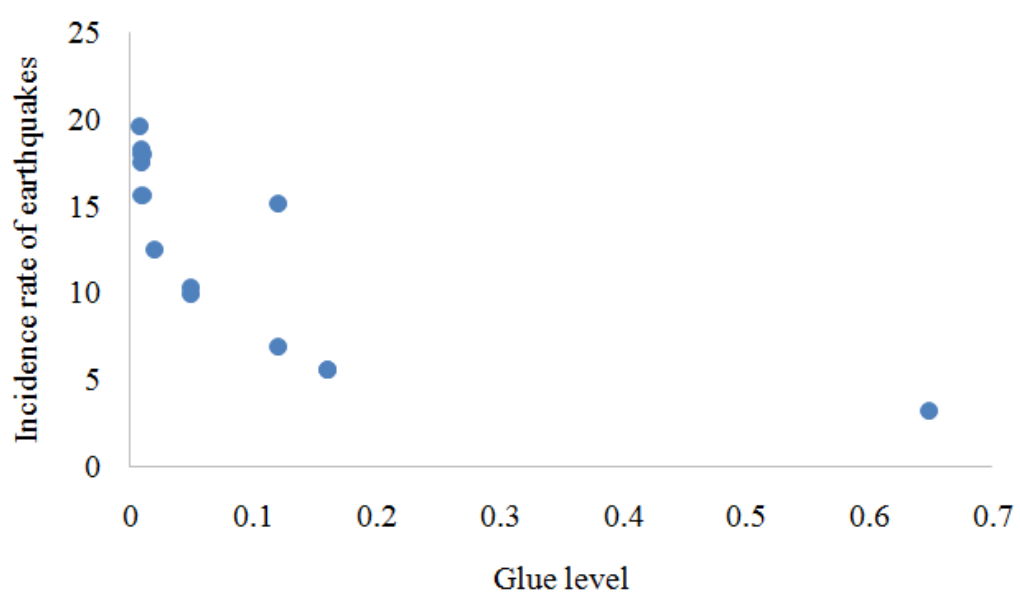

Fig. 5. Relation between the earthquakes and "glue" level

Among 13 tectonic plates

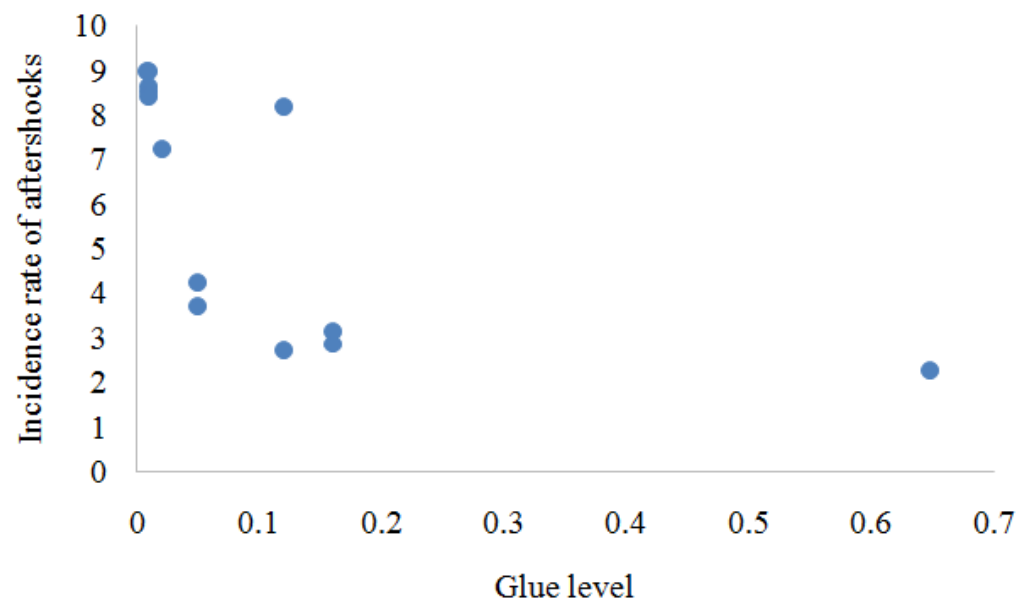

Fig. 6. Relation between aftershocks and the glue level

Among the tectonic plates

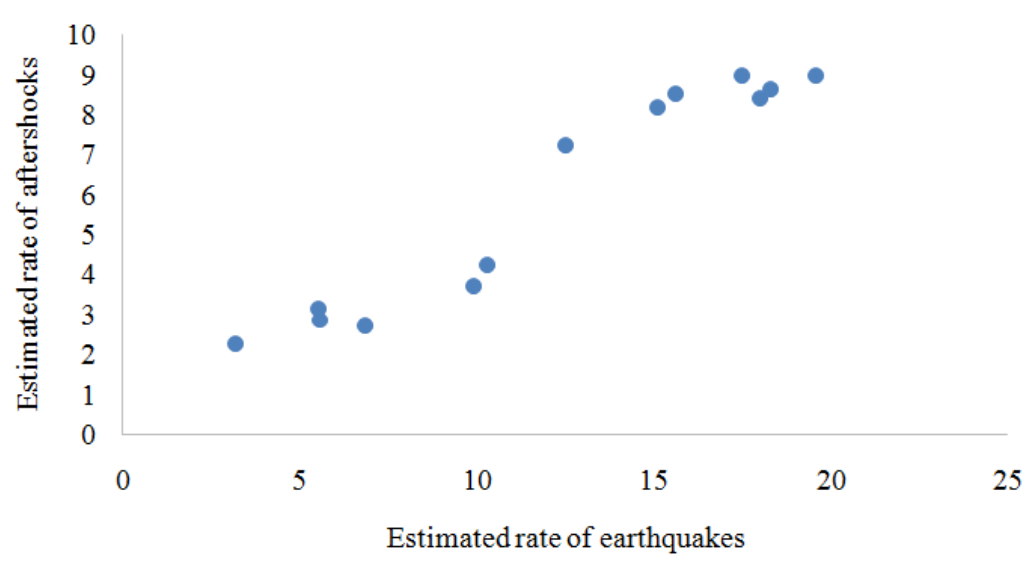

Fig. 7. Relation between the estimated earthquakes and aftershocks 


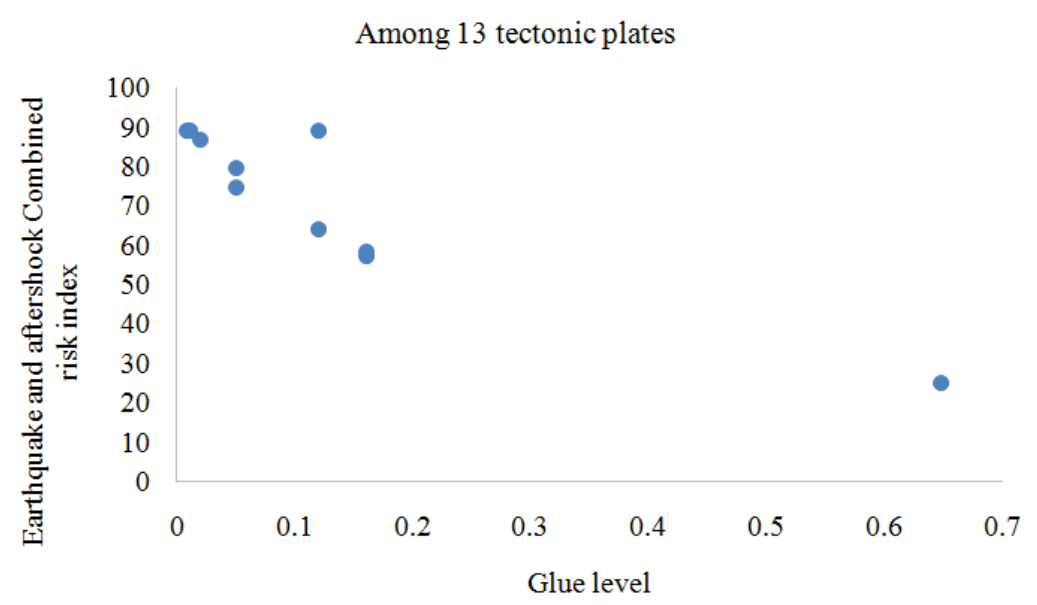

Fig. 8. Combined risk for earthquake or aftershock versus "glue" level

Table 2. A summary of the number (x) of earthquakes and the number (y) of aftershocks in and focused tectonic plates: AN = Antarctica, $\mathrm{AR}=$ Arabian, $\mathrm{AU}=$ Australia, $\mathrm{CA}=$ Caribbean, $\mathrm{CO}=\mathrm{Cocos}, \mathrm{EU}=$ Eurasian, $\mathrm{IN}=\mathrm{Indian}, \mathrm{NA}=\mathrm{North}$ American, $\mathrm{PA}=$ Pacific, $\mathrm{PH}=$ Philippines, $\mathrm{SA}=$ South American, $\mathrm{ST}=$ Scotia, and their adjacent plates, $\rho=\operatorname{corr}(X, Y)$

\begin{tabular}{|c|c|c|c|c|c|c|c|c|c|c|c|c|c|}
\hline Plates & $\mathrm{AF}$ & $\mathrm{AN}$ & AR & $\mathrm{AU}$ & $\mathrm{CA}$ & $\mathrm{CO}$ & EU & IN & NA & PA & $\mathrm{PH}$ & SA & ST \\
\hline$\rho_{(x, y)}$ & 0.200 & 0.200 & 0.300 & 0.300 & 0.700 & 0.400 & 0.400 & 0.200 & 0.100 & 0.200 & 0.300 & 0.100 & 0.100 \\
\hline$\hat{\phi}$ & 0.010 & 0.050 & 0.020 & 0.120 & 0.160 & 0.050 & 0.120 & 0.010 & 0.010 & 0.010 & 0.010 & 0.160 & 0.650 \\
\hline$\hat{\lambda}_{1}$ & 15.600 & 10.300 & 12.500 & 6.800 & 5.600 & 9.900 & 15.100 & 18.300 & 17.500 & 19.600 & 18.000 & 5.500 & 3.200 \\
\hline$\hat{\lambda}_{2}$ & 8.500 & 4.200 & 7.200 & 2.700 & 2.800 & 3.700 & 8.200 & 8.600 & 9.000 & 9.000 & 8.4 .00 & 3.100 & 2.200 \\
\hline $\mathfrak{R}_{e q}$ & 8.000 & 4.000 & 7.000 & 2.000 & 2.000 & 3.000 & 8.000 & 8.000 & 8.000 & 8.000 & 8.000 & 2.000 & 1.000 \\
\hline $\mathfrak{R}_{a s}$ & 643.000 & 177.000 & 112.000 & 24.000 & 7.000 & 183.000 & 553.000 & 7463.000 & 2620.000 & 18407.000 & 6894.000 & 6.000 & 1.000 \\
\hline$\theta$ eq $=\theta$ as $\approx$ & 1.571 & 1.571 & 1.571 & 1.571 & 1.571 & 1.571 & 1.571 & 1.571 & 1.571 & 1.571 & 1.571 & 1.571 & 1.571 \\
\hline $\mathfrak{I}$ & 89.000 & 80.000 & 87.000 & 64.000 & 58.000 & 75.000 & 89.000 & 89.000 & 89.000 & 89.000 & 89.000 & 57.000 & 25.000 \\
\hline
\end{tabular}

An intriguing relationship (Fig. 7) between the estimated aftershocks and earthquakes indicates that when one is more the other is also more. Analyzing one without the other is a futile exercise. Every data analysis or predictive warning ought to be done together the earthquakes and aftershocks.

Finally, it is worthwhile to construct a combined warning index for earthquake or aftershock to occur in each major tectonic plate. For this purpose, using the angles $(\angle A C B$ and $\angle A D B)$ in Trapezoid ABDC (Fig. 3 for details) and the data, a warning index, $\mathfrak{I}=100\left(\frac{\mathfrak{R}_{e q}}{1+\mathfrak{R}_{e q}}\right)\left(\frac{\mathfrak{R}_{a s}}{1+\mathfrak{R}_{a s}}\right)$ for the risk of having an earthquake or aftershock in each major tectonic plate could be constructed and it is summarized in (17). See Table 2 for the estimates of the risk index $\mathfrak{I}$ in a standardized scale $[0,1]$. The risk ranged from $25 \%$ (in Scotia plate) to $89 \%$ (in African, Eurasian, Indian, North American, Pacific and Philippines plates).

The estimated risk index, $\mathfrak{I}$ is sketched in terms of the estimated "glue" level of the major tectonic plates in Fig. 8. The pattern reveals an interesting lesson due to geometric approach that lesser the "glue" level among the adjacent plates refers a higher risk for earthquake or aftershock.

\section{Conclusion and Comments}

No wonder there was a major earthquake (with magnitude 7.3 in Rector Scale) and an aftershock in Kathmandu, Nepal recently on 26 April 2015, as the warning risk index is $\mathfrak{I}=89 \%$ according to this article. The Eurasian and Indian plates collided on each other while both plates have a risk index of $89 \%$ according to the findings of this article. On 30 May 2015 (just two days ago), the location Chichi-Shima, Japan experienced an earthquake with a magnitude 7.8 as its Pacific plate moved beneath the Philippine plate and this article quantified a warning risk index of $\mathfrak{I}=89 \%$ for both plates. The contents of this article via a geometric approach are validated already by the Kathmandu and Chichi-Shima earthquake incidences. An interesting geologic-constancy of 1.571 is noticed based on our statistical approach and an appropriate representative random sampling. The geometry does indeed demystify 
the pattern of earthquake and aftershocks. There is much more that can be done to fully understand what our beloved mother earth undergoes uninformed manner.

\section{Acknowledgment}

The author appreciates and thanks the Texas State University for a support with a Faculty Development Leave in spring semester, 2015 to perform the research work leading to this article.

\section{Ethics}

This article is original and contains unpublished material. The corresponding author confirms that all of the other authors have read and approved the manuscript and no ethical issues involved.

\section{References}

Abe, S. and N. Suzuki, 2007. Event-event correlation in seismicity and aging of aftershocks. AAPPS Bull., 17: 27-29.

Berkhout, P. and E. Plug, 2004. A bivariate Poisson count data model using conditional probabilities. Statist. Neerlandica, 58: 349-364. DOI: $10.1111 / \mathrm{j} .1467-9574.2004 .00126 . x$

Beroza, G.C., 2012. How many great earthquakes should we expect? Proc. Nati. Acad. Sci. USA., 109: 651-652. DOI: 10.1073/pnas.1120744109

Felzer, K.R. and E.E. Brodsky, 2006. Decay of aftershock density with distance indicates triggering by dynamic stress. Nature, 441: 735-738. DOI: 10.1038 /nature04799

Helmstetter, A., Y.Y. Kagan and D.D. Jackson, 2005. Importance of small earthquakes for stress transfers and earthquake triggering. J. Geophys. Res., 110: B05S08-B05S08. DOI: 10.1029/2004JB003286

http://en.wikipedia.org/List_of_2 $1^{\text {st }}$ century_earthquakes.

Johnson, N., S. Kotz and N. Balakrishnan, 1997. Discrete Multivariate Distributions. 1st Edn., Wiley, New York, ISBN-10: 0471128449, pp: 328.

Marekova, E., 2014. Analysis of the spatial distribution between successive earthquakes in aftershocks series. Ann. Geophys., 57: 1-12.

DOI: $10.4401 /$ ag-6556
Métivier, L., O. de Viron, C. Conrad, S. Renault and M. Diament et al., 2009. Evidence of earthquake triggering by the solid earth tides. Earth Planet. Sci. Lett., 278: 370-375. DOI: $10.1016 /$ j.epsl.2008.12.024

Ogata, Y., 2001. Increased probability of large earthquakes near aftershock regions with relative quiescence. J. Geophys. Res., 106: 8720-8744. DOI: $10.1029 / 2000 J B 900400$

Polat, O., H. Eyidogan, H. Haessler, A. Cisternas and H. Philip, 2002. Analysis and interpretation of the aftershock sequence of the August 17, 1999, Izmit (Turkey) earthquake. J. Seismol., 6: 287-206. DOI: $10.1023 / \mathrm{A}: 1020075106875$

Richter, C.F., 1958. Elementary Seismology. 1st Edn., W. H. Freeman, San Francisco, pp: 768.

Rubin, A.M., 2002. Aftershocks of micro earthquakes as probes of the mechanics of rupture. J. Geophys. Res., 107: ESE 3-1-ESE 3-16. DOI: $10.1029 / 2001 J B 000496$

Schoenberg, F.P., C. Barr and J. Seo, 2008. The distribution of Voronoi cells generated stress. J. Geophys. Res., 103: 24453-24267.

Shcherbakov, R. and D.L. Turcotte, 2004. A damage mechanics model for aftershocks. Pure Applied Geophys., 161: 2379-2391. DOI: $10.1007 / \mathrm{s} 00024-004-2570-\mathrm{x}$

Shcherbakov, R., D.L. Turcotte and J.B. Rundle, 2005. Aftershock statistics. Pure Applied Geophys., 165: 1051-1076. DOI: 10.1007/s00024-004-2661-8

Shcherbakov, R., J.V. Aalsburg, J.B. Rundle and D.L. Turcotte, 2006. Correlations in aftershock and seismicity patterns. Tectonophysics, 413: 53-62. DOI: $10.1016 /$ j.tecto.2005.10.009

Shearer, P.M. and P.B. Stark, 2012. Global risk of big earthquakes has not recently increased. Proc. Nat. Acad. Sci. USA., 109: 717-721.

Wegener, A., 1912. Die Herausbildung der Grossformen der Erdrinde (Kontinente und Ozeane), auf geophysikalischer Grundlage. Petermanns Geographische Mitteilungen, 63: 185-195. 